\title{
A case for expert curation: an overview of cancer curation in the Clinical Genome Resource (ClinGen)
}

\author{
Deborah I. Ritter, ${ }^{1,2}$ Shruti Rao, ${ }^{3}$ Shashikant Kulkarni, ${ }^{1,4}$ Subha Madhavan, ${ }^{3}$ \\ Kenneth Offit, ${ }^{5,6}$ and Sharon E. Plon ${ }^{1,2}$ \\ ${ }^{1}$ Baylor College of Medicine, Houston, Texas 77030, USA; ${ }^{2}$ Texas Children's Cancer Center, Texas Children's \\ Hospital, Houston, Texas 77030, USA; ${ }^{3}$ Innovation Center for Biomedical Informatics, Georgetown University, \\ Washington, D.C. 20007, USA; ${ }^{4}$ Baylor Genetics, Houston, Texas 77021, USA; ${ }^{5}$ Department of Medicine, \\ Memorial Sloan Kettering Cancer Center, New York, New York 10065, USA; ${ }^{6}$ Sloan Kettering Institute, \\ New York, New York 10065, USA
}

Abstract We describe the Clinical Genome Resource (ClinGen) cancer-related curation activities and the importance of curation to the evolving state of variant interpretation in a clinical context for both pediatric and adult cancer patients. We highlight specific examples from the CDH1 and PTEN Variant Curation Expert Panels (VCEPs) of the FDA-recognized process by which ClinGen VCEPs specify the American College of Medical Genetics and Genomics/Association of Molecular Pathology evidence code to develop variant classifications. We also review gene curations performed within the Hereditary Cancer Clinical Domain. We describe the parallel efforts for curation of somatic cancer variants from the Somatic Cancer Working Group. The ClinGen Germline/Somatic Committee is working to improve incorporation of both hereditary and somatic variant data to aid clinical interpretation. These ClinGen efforts rely on broad data sharing and detailed phenotypic and molecular information from published case studies to provide expert-curated variant interpretation to the cancer community.

Corresponding author: splon@bcm.edu

(C) 2019 Ritter et al. This article is distributed under the terms of the Creative Commons Attribution-NonCommercial License, which permits reuse and redistribution, except for commercial purposes, provided that the original author and source are credited.

Published by Cold Spring Harbor Laboratory Press

doi:10.1101/mcs.a004739

\section{INTRODUCTION}

The clinical use of DNA sequencing has steadily increased as the price point declines, test options expand, and turnaround times decrease. However, for the sequenced genome to be of any clinical use, we must understand the function of genes and the impact of variants in relation to human diseases-the challenge of interpreting the genome. Curation of the human genome is a time-consuming process that relies on information about genes and variants extracted from a variety of diverse unstructured sources-published literature, collections of laboratory clinical data, disease-specific groups, and databases of functional experiments-to generate a clinical variant interpretation as specified in the American College of Medical Genetics and Genomics/Association of Molecular Pathology (ACMG/ AMP) guidelines (Richards et al. 2015). This special issue emphasizes the utility of curation and variant interpretation of both somatic and germline variants associated with childhood cancer. We briefly describe here the efforts within The Clinical Genome Resource (ClinGen) to increase the availability of expert-curated knowledge relevant to hereditary and somatic cancers. As pediatric cancers are often at the interface of hereditary genetics and somatic tumor variants, variant interpretation in these complex cases can greatly benefit from an approach that organizes expert-level insight. 
ClinGen (clinicalgenome.org) is a multi-institutional effort to standardize gene and variant curation, advance the utility of genomics in medicine, and develop and standardize the process by which genomic variants are interpreted by highly qualified individuals in science and medicine (Rehm et al. 2015). To focus curation and standardization efforts on realms most in need of clarification, ClinGen gene and variant curation is currently organized into eight Clinical Domain Working Groups (CDWG): Cardiovascular, Hearing Loss, Hemostasis/ Thrombosis, Hereditary Cancer, Inborn Errors of Metabolism, Neurodevelopmental, Neuromuscular, and RASopathies. Each CDWG oversees multiple Gene or Variant Curation Expert Panels (GCEPs or VCEPs) performing curation activities on gene sets and diseases within the domain umbrella (Rivera-Muñoz et al. 2018). In a coordinated effort with the U.S. Food and Drug Administration (FDA), ClinGen is the first FDA-recognized genetic variant information source. Per the FDA's guidance document, Use of Public Human Genetic Variant Databases to Support Clinical Validity for Genetic and Genomic-Based In Vitro Diagnostics, the FDA recognized that the ClinGen variant curation expert panel variant classification process provided "reasonable assurance that the variant assertions made were accurate and could be used as a source of valid scientific evidence in support of clinical validity of genetic and genomic-based tests in regulatory submissions" (https://www.fda.gov/media/119313/download). The process by which VCEPs form, curate, submit interpreted variants, and regularly update these classifications was developed in communication with the FDA to create a standardized and transparent mechanism for ensuring curation quality, history, and fidelity ( $\mathrm{O}^{\prime}$ Daniel and Berg 2016).

There are several additional ClinGen working groups that serve in guidance roles for interpretation efforts. The Sequence Variant Interpretation Working Group (SVI) has provided guidance for implementation of ACMG/AMP evidence codes as well as features that may benefit from modification based on gene specificity (Richards et al. 2015; Abou Tayoun et al. 2018). The SVI serves as a governing body to review and approve VCEP gene-specific criteria, which ultimately provide precise and nuanced information to aid and improve variant interpretations. The Clinical Domain Oversight Committee confers final approval prior to publication and submission of classified variants by the ClinGen VCEP to ClinVar, a public variant database hosted by the National Library of Medicine (Landrum and Kattman 2018).

\section{HEREDITARY CANCER IN THE CLINICAL GENOME RESOURCE}

Although ClinGen is largely focused on Mendelian conditions, there is a sizable effort in curating cancer susceptibility genes and variants, as well as those involved in somatic variation (Fig. 1). The Hereditary Cancer CDWG identified the initial genes to develop hereditary cancer VCEPs through a needs assessment presented by Rivera-Muñoz at the 2016 ACMG Annual Meeting. The assessment prioritized hereditary cancer genes without existing variant curation efforts (i.e., Evidence-based Network for the Interpretation of Germline Mutant Alleles [ENIGMA] for BRCA1/BRCA2 and International Society for Gastrointestinal Hereditary Tumors [InSiGHT] for mismatch repair genes), estimated cancer risk associated with pathogenic variants from literature review, ranked the number of conflicting and uncertain variants in ClinVar, prioritized genes included on the ACMG Secondary Findings list, and assessed the presence on hereditary cancer sequencing panels among seven large clinical sequencing laboratories (Kalia et al. 2017). This process identified genes, including CDH1, TP53, and PTEN, that may have implications in both adult and pediatric cancers as high priority targets. The PTEN and CDH1 VCEPs were initiated first and approved in late 2018 (Lee et al. 2018; Mester et al. 2018) and are actively submitting curated variants to ClinVar, with TP53 nearly complete. Subsequently, additional VCEPs were initiated by the Hereditary Cancer CDWG with now seven VCEPs, focusing on Myeloid Malignancies (in a collaboration 


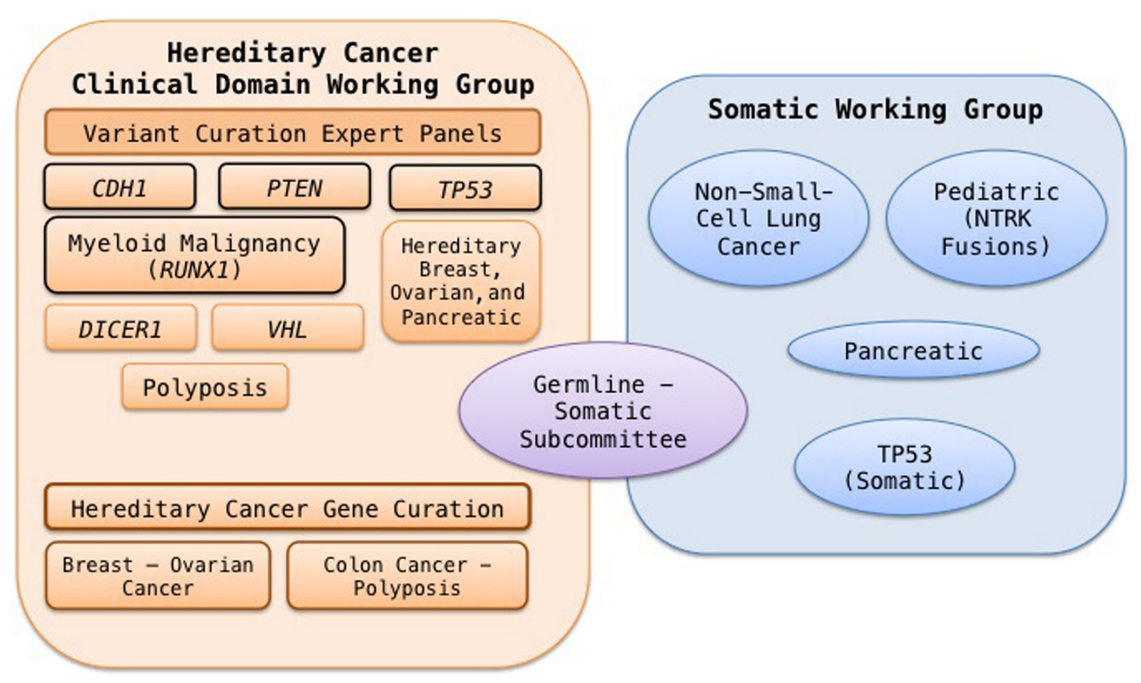

Figure 1. The Clinical Genome Resource cancer efforts. Approved ClinGen Variant Curation Expert Panels (VCEPs) are outlined in bold. Gene curation is outlined in red. As described in the text, many of the VCEPs operate in collaboration with external organizations (not shown here).

with the American Society of Hematology, RUNX1), VHL, DICER1, and Hereditary Breast/ Ovarian and Pancreatic Cancers (in collaboration with ENIGMA), as well as PTEN, CDH1, and TP53. In addition, a Polyposis VCEP through a joint effort with the International Society for Gastrointestinal Hereditary Tumors (InSiGHT) is being developed.

Results of these ClinGen VCEPs are highly relevant to variant interpretations in pediatric cancers, as the variants in the genes under study can manifest in childhood cancer susceptibility conditions such as Li-Fraumeni (TP53), myeloid leukemias (RUNX1), and PTEN hamartoma tumor syndromes and pulmonary pleuroblastoma (DICER1). Through clarification and specification of the ACMG/AMP Variant Interpretation Guidelines, curation of variants in the ClinGen Variant Curation Interface (VCI), and expert review, the VCEPs can provide interpreted variant sets to ClinVar and the ClinGen evidence repository (eRepo; erepo.clinicalgenome.org), encourage data sharing from diagnostic laboratories, create consensus interpretations, and develop a compendium of gene-specified ACMG/AMP evidence codes that can ultimately ensure more accurate interpretations for clinical use. A brief overview of recent relevant findings from PTEN and CDH1 VCEPs will serve to highlight their implications for variant interpretation, and the contribution VCEP interpretations can make to resources such as ClinVar (Lee et al. 2018; Mester et al. 2018).

An example of the novel development and detailed contribution VCEPs can make to variant interpretation is found in the PTEN VCEP creation of a pediatric PTEN phenotype specificity scoring system (see Table 2 in Mester et al. 2018) for use with the PS4/PP4 evidence codes which handle disease-specific phenotypic data. This is modeled after the Cleveland Clinic (CC) score for adult PTEN cases and built from pooled, de-identified pediatric PTEN cohorts shared between U.S. and U.K. laboratories (Tan et al. 2011). The careful delineation of PTEN pediatric-specific phenotypic data to build this scoring tool will create greater consistency in pediatric PTEN variant interpretations. Perhaps more than other evidence codes, the phenotypic codes underscore the importance of case study publications such as in Molecular Case Studies and the sharing of laboratory cohort data in the Expert Panel process.

The PTEN and CHD1 VCEPs have each substantially modified the ACMG BA1/BS1 benign evidence codes from the published default for BA1 stand-alone benign code 
$(\geq 5 \%)$ to frequency cutoffs that reflect the disease incidence. Having disease-specific cutoffs for rare variants in control databases such as ExAC and gnomAD will create more consistency in variant interpretation for benign and likely benign categories. For example, the $\mathrm{CDH} 1$ VCEP used an estimation of hereditary diffuse gastric cancer incidence and accounted for an estimated penetrance of $\sim 30 \%$ for $\mathrm{CDH} 1$ variants, as well as considered the incidence of lobular breast cancer in affected families. These elements factored into the calculation of BA1 allele frequency of $\geq 0.002(0.2 \%)$ and BS1 of $\geq 0.001(0.1 \%)$. In addition, the calculations, reasoning, and methods VCEPs use to establish BA1/BS1 provide examples for other laboratories interpreting variants to carefully consider appropriate BA1/BS1 rather than use the default of $5 \%$; this increases the number of variants that can be classified Benign through the use of these gene-specified codes.

Although many of the published ClinGen VCEPs have identified functional data sets (PS3 evidence code) for variant interpretation, CDH1 VCEP opted to advise against using functional data to support interpretations of $\mathrm{CDH1}$ missense variants. This surprising finding came after a thorough literature search did not reveal published in vitro or in vivo E-cadherin functional studies that could be confidently used to predict missense variant pathogenicity. Likewise, for computational predictions (PP3 evidence code), the CDH1 VCEP tested many in silico algorithms (REVEL, VEST3, MetaSVM, CADD, SIFT, PolyPhen-2, and Provean) and found none to reliably interpret missense $\mathrm{CDH} 1$ variants. Both the computational and functional outcomes will have a substantial impact on increasing consistency in $\mathrm{CDH} 1$ variant interpretations.

A primary contribution for any VCEP is the interpretive resolution of "problematic" variants-variants that have multiple, conflicting, or variant of uncertain significance (VUS) submissions in ClinVar and need an expert review to adjudicate. An example is NM_ 000314.7(PTEN):c.235G>A (p.Ala79Thr). In ClinVar the variant had conflicting interpretations, with 10 VUSs, one Pathogenic, and three Likely Benign. The PTEN VCEP interpretation after applying the PTEN specifications is Likely Benign. This expert panel Likely Benign interpretation can help families that may carry this variant avoid considerable stress and uncertainty. When comparing the interpretations of the PTEN VCEP to that of all PTEN variants in ClinVar, there are about one-half the proportion of VUSs (31/111 28\% PTEN VCEP vs. 719/ $1585 \sim 45 \%$ all PTEN variants). Notably, this comparison is slightly biased because of the inclusion of known benign/pathogenic variants to develop and test evidence code criteria; however, this is reinforced by the VUS resolution achieved by the CDH1 VCEP, wherein they applied the CDH1-specified evidence codes to 20 VUSs (12 submitted by VCEP members and eight selected from ClinVar) and reduced VUS classifications from 20 to 11 ( 45\%). We expect a major, lasting impact and effort of VCEPs will be to resolve VUS classification, particularly those reported by multiple different laboratories as VUS (by allowing the VCEP to combine this existing data) and those with conflicting interpretations of pathogenicity in ClinVar.

Indeed, VCEP interpretations are already increasing consistency and displaying influence over other submissions in ClinVar as submitters incorporate the VCEP interpretation into their own variant evaluation. An example is PTEN c.209+4_209+7delAGTA (IVS3+4_IVS3 +7delAGTA); this variant had two Pathogenic and one VUS when first evaluated by the PTEN VCEP as Pathogenic (10-29-17). It currently has three Pathogenic and one Likely Pathogenic; the VUS interpretation was modified or removed. Similarly, shortly after the $\mathrm{CDH} 1$ publication, a ClinVar submission referenced the published CDH1 VCEP interpretation for NM_004360.5(CDH1):c.2494G>A (p.Val832Met) as benign. This CDH1 variant has a long history of conflicting interpretations. The quick reference in a new submission speaks to the strong influence ClinGen VCEP interpretations will have and ultimately their impact on the clinical utility of sequenced variants.

In addition to variant curation, the Hereditary Cancer Clinical Domain engages in gene curation, using the ClinGen gene curation process to describe the strength of genetic and 
functional evidence that variation in a gene is associated with a Mendelian phenotype (with some modifications for cancer curation) (Strande et al. 2017). Gene curation will likely have the greatest impact on the composition of hereditary cancer gene testing panels. For example, the Colorectal Cancer/Polyposis Gene Curation Working Group identified BLM as a Definitive Colorectal Cancer/Polyposis gene, however, at the time of publication (November 2018) it was only offered on three hereditary Colorectal/Polyposis testing panels (Seifert et al. 2019). In the Breast/Ovarian Gene Curation Working Group, nine of 31 genes ( 29\%) found on breast/ovarian hereditary cancer panels and evaluated for Breast Cancer susceptibility received Refuted or Disputed curation classifications: MLH1, PMS2, MSH6, MSH2, RINT1, RAD51C, MRE11A, BRIP1, and GEN1 (Lee et al. 2019). Gene curations performed in this standardized and transparent process can help to clarify and strengthen the content of gene panels. In addition, the Hereditary Cancer Gene Curation Working Group is systematically curating all of the cancer predisposition genes described in Rahman (2014) in the ClinGen Gene Curation Interface with the results published to www .clinicalgenome.org.

\section{SOMATIC CANCER IN THE CLINICAL GENOME RESOURCE}

Pediatric cancers often develop from a combination of both hereditary and acquired (tumor) variants, as proposed by Knudson's two-hit hypothesis (Knudson 2001). However, with expanded cohorts of pediatric cancer sequencing, additional unexpected overlap between germline and somatic variation (as well as pediatric and adult cancers) has been seen; for example, EGFR germline mutations seen in pediatric leukemia were also known activating somatic "hotspot" mutations (at codons T790 and H773) in adult cancers (Zhang et al. 2015; Parsons et al. 2016). Recently, the association of BRCA2 variants typically associated with germline variation in adult cancers have also been reported in pediatric lymphoma (Wang et al. 2019). In response, ClinGen developed the Somatic Cancer Working Group (WG), which focuses on somatic variant curation and standardization, as well as the Germline/Somatic Variant Subcommittee, which develops recommendations and points to consider at the interface of somatic and germline cancers (Walsh et al. 2018).

As a first step, the Somatic Cancer WG developed a cancer variant curation structure termed Minimum Variant Level Data (MVLD) to describe a structured way to convey variants found in cancer analyses (Ritter et al. 2016). Subsequently, the somatic variant interpretation guidelines were published by the Association for Molecular Pathology (AMP), and MLVD was adjusted to match this framework, incorporating the AMP guidelines into the "Level of Evidence" field (Li et al. 2017; Danos et al. 2018).

Following this, ClinGen has formed task teams for curation efforts in Pancreatic, TP53, Pediatric Cancers, and Lung Cancers. The somatic curation efforts intend to develop an expert panel process modeled after the germline ClinGen VCEPs. These Cancer Curation Expert Panels (CCEPs) will further clarify and specify the somatic variant interpretation guidelines by providing expert interpretations and focused curation to areas of need. NTRK fusions in pediatric cancers have been chosen as a first clinically relevant example for developing the CCEP model with others to follow. The NTRK work emphasizes the need to develop standardization of fusion terminology particularly for fusions that impact treatment. The group will be developing a workflow for identifying NTRK fusion literature and cases, extracting relevant information to an MVLD fusion format, and assigning biocurators to add Evidence Items within the Clinical Interpretations of Variants in Cancer (CIViC, see below) database. The group will involve expert review to create "Assertions" within CIViC based on the AMP Somatic Interpretation guidelines. 
COLD SPRING HARBOR Molecular Case Studies
A case for expert curation in cancer
Competing Interest Statement

S.M. is an advisor/consultant to Perthera Inc. and CRC Alliance. Her research team at Georgetown University received (or currently receives) funding from the National Institutes of Health (NIH), the Food and Drug Administration (FDA), and the Office of the National Coordinator for Health Information Technology (ONC). She is the co-founder of Health IT consulting firm Technology for Science, LLC. S.K. is Chief Scientific Officer of Baylor Genetics. S.E.P. is a member of the scientific advisory panel for Baylor Genetics.
The modeling of cancer variant data to harmonize and allow searchability across multiple data sets has been a focus for many efforts, including the GA4GH Variant Interpretation in Cancer Consortium (VICC) subgroup as well as large knowledgebases for cancer variants including Jackson Laboratories Clinical Knowledgebase, OncoKB, and CIViC. In an attempt to harness and standardize literature on cancer case studies, journals such as Molecular Case Studies, Cancer Genetics (now using the MVLD somatic format), and others are prioritizing rapid case publications with streamlined processes to ensure variants are deposited in public databases such as ClinVar and CIViC. CIViC (www.civicdb.org) is a public cancer variant database that allows researchers and clinicians to curate variants in a distributed, asynchronous manner (Griffith et al. 2017). In parallel to germline curation in the ClinGen interface, variant curation from the Somatic Working Group CCEPs will be performed in $\mathrm{CIViC}$, and in turn, $\mathrm{CIViC}$ will make submissions to ClinVar.

The ClinGen Germline/Somatic Variant Subcommittee (GSVS) efforts are highly relevant to pediatric cancers, as many have underlying germline susceptibility but may also harbor somatic or sporadic tumor variants in the same genes. This group has recently published a suggested methodology to incorporate existing somatic data from cancer sequencing studies into the interpretation of germline variants (Walsh et al. 2018). The interest to incorporate somatic data was driven by pediatric cases in which germline variants in hereditary cancer patients lack supporting germline hotspot data but are found at some level of prevalence in somatic tumor databases such as cancerhotspots.org (Chang et al. 2016, 2018). The GSVS provided specific recommendations to use somatic data in the ACMG PM1 "hotspot" evidence code (Walsh et al. 2018). The TP53 VCEP, as well as the VHL VCEP is adopting this PM1 recommendation. VCEPs can evaluate somatic cancer data for their gene to assess the impact of incorporating somatic data to support PM1, following the guidelines and resources outlined. In addition, the GSVS has provided examples of other evidence codes in which somatic data may be considered, such as tumor profiling as a phenotypic feature. The GSVS continues to work on the interplay of classification for hereditary and somatic variants.

The model of expert panels providing variant interpretations and gene-specific guidance on variant interpretation is likely to become an integral part of the future of variant interpretation, with a significant impact for clinical cancer genetics and ultimately for medical decision-making in pediatric cancer cases. It will be important to identify trends in decision-making and specifications and to create formats to share and integrate the products of ClinGen Expert Panels, especially into tools that automate variant interpretations to decrease the time and resource burden on diagnostic laboratories. Although expert curation contains manual review and can become time-consuming work, ongoing efforts to crowdsource some elements of curation such as literature search, knowledge gained from the structured molecular and detailed phenotypic data contained in Molecular Case Studies, and the ongoing collaboration of large testing laboratories with ClinGen processes will continue to increase the pace of curation outputs. In addition, research on machine-learning approaches such as Natural Language Processing to use curated evidence from VCEPs to train curation models and deep-learning methods to predict outcomes will be fundamental to increasing the throughput of the clinical variant interpretation process (Mahmood et al. 2017). The scaled organization of expert insight into variant interpretation will ultimately achieve a more robustly curated genome with enhanced clinical utility.

\section{ADDITIONAL INFORMATION}

\section{Acknowledgments}

The authors thank the leadership of Erin Ramos, National Human Genome Research Institute, the ClinGen Steering Committee, and the many collaborating organizations in ongoing support of these ClinGen cancer efforts. 


\section{Author Contributions}

All authors contributed substantially to the work described.

\section{Funding}

ClinGen is primarily funded by the National Human Genome Research Institute (NHGRI), through the following three grants: U41HG006834, U41HG009649, and U41HG009650 (and formerly U01HG007436, U41HG009650, and U01HG007437). The content is solely the responsibility of the authors and does not necessarily represent the official views of the National Institutes of Health.

\section{REFERENCES}

Abou Tayoun AN, Pesaran T, DiStefano MT, Oza A, Rehm HL, Biesecker LG, Harrison SM, ClinGen Sequence Variant Interpretation Working Group (ClinGen SVI). 2018. Recommendations for interpreting the loss of function PVS1 ACMG/AMP variant criterion. Hum Mutat 39: 1517-1524. doi:10.1002/humu.23626

Chang MT, Asthana S, Gao SP, Lee BH, Chapman JS, Kandoth C, Gao J, Socci ND, Solit DB, Olshen AB, et al. 2016. Identifying recurrent mutations in cancer reveals widespread lineage diversity and mutational specificity. Nat Biotechnol 34: 155-163. doi:10.1038/nbt.3391

Chang MT, Bhattarai TS, Schram AM, Bielski CM, Donoghue MTA, Jonsson P, Chakravarty D, Phillips S, Kandoth C, Penson A, et al. 2018. Accelerating discovery of functional mutant alleles in cancer. Cancer Discov 8: 174-183. doi:10.1158/2159-8290.CD-17-0321

Danos AM, Ritter DI, Wagner AH, Krysiak K, Sonkin D, Micheel C, McCoy M, Rao S, Raca G, Boca SM, et al. 2018. Adapting crowdsourced clinical cancer curation in CIViC to the ClinGen minimum variant level data community-driven standards. Hum Mutat 39: 1721-1732. doi:10.1002/humu.23651

Griffith M, Spies NC, Krysiak K, McMichael JF, Coffman AC, Danos AM, Ainscough BJ, Ramirez CA, Rieke DT, Kujan $\mathrm{L}$, et al. 2017. CIViC is a community knowledgebase for expert crowdsourcing the clinical interpretation of variants in cancer. Nat Genet 49: 170-174. doi:10.1038/ng.3774

Kalia SS, Adelman K, Bale SJ, Chung WK, Eng C, Evans JP, Herman GE, Hufnagel SB, Klein TE, Korf BR, et al. 2017. Recommendations for reporting of secondary findings in clinical exome and genome sequencing, 2016 update (ACMG SF v2.0): a policy statement of the American College of Medical Genetics and Genomics. Genet Med 19: 249-255. doi:10.1038/gim.2016.190

Knudson AG. 2001. Two genetic hits (more or less) to cancer. Nat Rev Cancer 1: 157-162. doi:10.1038/ 35101031

Landrum MJ, Kattman BL. 2018. ClinVar at five years: delivering on the promise. Hum Mutat 39: 1623-1630. doi:10.1002/humu.23641

Lee K, Seifert BA, Shimelis H, Ghosh R, Crowley SB, Carter NJ, Doonanco K, Foreman AK, Ritter DI, Jimenez S, et al. 2019. Clinical validity assessment of genes frequently tested on hereditary breast and ovarian cancer susceptibility sequencing panels. Genet Med 21: 1497-1506. doi:10.1038/s41436-018-0361-5

Lee K, Krempely K, Roberts ME, Anderson MJ, Carneiro F, Chao E, Dixon K, Figueiredo J, Ghosh R, Huntsman $D$, et al. 2018. Specifications of the ACMG/AMP variant curation guidelines for the analysis of germline CDH1 sequence variants. Hum Mutat 39: 1553-1568. doi:10.1002/humu.23650

Li MM, Datto M, Duncavage EJ, Kulkarni S, Lindeman NI, Roy S, Tsimberidou AM, Vnencak-Jones CL, Wolff DJ, Younes A, et al. 2017. Standards and guidelines for the interpretation and reporting of sequence variants in cancer: a joint consensus recommendation of the Association for Molecular Pathology, American Society of Clinical Oncology, and College of American Pathologists. J Mol Diagn 19: 4-23. doi:10 .1016/j.jmoldx.2016.10.002

Mahmood ASMA, Rao S, McGarvey P, Wu C, Madhavan S, Vijay-Shanker K. 2017. eGARD: extracting associations between genomic anomalies and drug responses from text. PLoS One 12: e0189663. doi:10.1371/ journal.pone.0189663

Mester JL, Ghosh R, Pesaran T, Huether R, Karam R, Hruska KS, Costa HA, Lachlan K, Ngeow J, Barnholtz-Sloan $J$, et al. 2018. Gene-specific criteria for PTEN variant curation: recommendations from the ClinGen PTEN expert panel. Hum Mutat 39: 1581-1592. doi:10.1002/humu.23636

O'Daniel JM, Berg JS. 2016. A missing link in the bench-to-bedside paradigm: engaging regulatory stakeholders in clinical genomics research. Genome Med 8: 95. doi:10.1186/s13073-016-0351-7

Parsons DW, Roy A, Yang Y, Wang T, Scollon S, Bergstrom K, Kerstein RA, Gutierrez S, Petersen AK, Bavle A, et al. 2016. Diagnostic yield of clinical tumor and germline whole-exome sequencing for children with solid tumors. JAMA Oncol 2: 616-624. doi:10.1001/jamaoncol.2015.5699 
Rahman N. 2014. Realising the promise of cancer predisposition genes. Nature 505: 302-308. doi:10.1038/ nature12981

Rehm HL, Berg JS, Brooks LD, Bustamante CD, Evans JP, Landrum MJ, Ledbetter DH, Maglott DR, Martin CL, Nussbaum RL, et al. 2015. ClinGen—the clinical genome resource. N Engl J Med 372: 2235-2242. doi:10 .1056/NEJMsr1406261

Richards S, Aziz N, Bale S, Bick D, Das S, Gastier-Foster J, Grody WW, Hegde M, Lyon E, Spector E, et al. 2015. Standards and guidelines for the interpretation of sequence variants: a joint consensus recommendation of the American College of Medical Genetics and Genomics and the Association for Molecular Pathology. Genet Med 17: 405-424. doi:10.1038/gim.2015.30

Ritter DI, Roychowdhury S, Roy A, Rao S, Landrum MJ, Sonkin D, Shekar M, Davis CF, Hart RK, Micheel C, et al. 2016. Somatic cancer variant curation and harmonization through consensus minimum variant level data. Genome Med 8: 117. doi:10.1186/s13073-016-0367-z

Rivera-Muñoz EA, Milko LV, Harrison SM, Azzariti DR, Kurtz CL, Lee K, Mester JL, Weaver MA, Currey E, Craigen W, et al. 2018. ClinGen variant curation expert panel experiences and standardized processes for disease and gene-level specification of the ACMG/AMP guidelines for sequence variant interpretation. Hum Mutat 39: 1614-1622. doi:10.1002/humu.23645

Seifert BA, McGlaughon JL, Jackson SA, Ritter DI, Roberts ME, Schmidt RJ, Thompson BA, Jimenez S, Trapp M, Lee K, et al. 2019. Determining the clinical validity of hereditary colorectal cancer and polyposis susceptibility genes using the clinical genome resource clinical validity framework. Genet Med 21: 15071516. doi:10.1038/s41436-018-0373-1

Strande NT, Riggs ER, Buchanan AH, Ceyhan-Birsoy O, DiStefano M, Dwight SS, Goldstein J, Ghosh R, Seifert BA, Sneddon TP, et al. 2017. Evaluating the clinical validity of gene-disease associations: an evidencebased framework developed by the clinical genome resource. Am J Hum Genet 100: 895-906. doi:10 .1016/j.ajhg.2017.04.015

Tan MH, Mester J, Peterson C, Yang Y, Chen JL, Rybicki LA, Milas K, Pederson H, Remzi B, Orloff MS, et al. 2011. A clinical scoring system for selection of patients for PTEN mutation testing is proposed on the basis of a prospective study of 3042 probands. Am J Hum Genet 88: 42-56. doi:10.1016/j.ajhg.2010.11.013

Walsh MF, Ritter DI, Kesserwan C, Sonkin D, Chakravarty D, Chao E, Ghosh R, Kemel Y, Wu G, Lee K, et al. 2018. Integrating somatic variant data and biomarkers for germline variant classification in cancer predisposition genes. Hum Mutat 39: 1542-1552. doi:10.1002/humu.23640

Wang Z, Wilson CL, Armstrong GT, Hudson MM, Zhang J, Nichols KE, Robison LL. 2019. Association of germline BRCA2 mutations with the risk of pediatric or adolescent non-Hodgkin lymphoma. JAMA Oncol 5: 1362-1364. doi: 10.1001/jamaoncol.2019.2203.

Zhang J, Walsh MF, Wu G, Edmonson MN, Gruber TA, Easton J, Hedges D, Ma X, Zhou X, Yergeau DA, et al. 2015. Germline mutations in predisposition genes in pediatric cancer. N Engl J Med 373: 2336-2346. doi:10.1056/NEJMoa1508054 


\section{COLD SPRING HARBOR Molecular Case Studies}

\section{A case for expert curation: an overview of cancer curation in the Clinical Genome Resource (ClinGen)}

Deborah I. Ritter, Shruti Rao, Shashikant Kulkarni, et al.

Cold Spring Harb Mol Case Stud 2019, 5: a004739

Access the most recent version at doi: $10.1101 / \mathrm{mcs} . a 004739$

References This article cites 26 articles, 1 of which can be accessed free at: http://molecularcasestudies.cshlp.org/content/5/5/a004739.full.html\#ref-list-1

License This article is distributed under the terms of the Creative Commons Attribution-NonCommercial License, which permits reuse and redistribution, except for commercial purposes, provided that the original author and source are credited.

Email Alerting Receive free email alerts when new articles cite this article - sign up in the box at the Service top right corner of the article or click here. 